\title{
New NCCN Guidelines: Cancer Management in People Living With HIV
}

\author{
Presented by Gita Suneja, MD, MSHP
}

\begin{abstract}
Since the emergence of HIV in the United States in the 1980s, cancer has been a part of the story. The good news is that antiretroviral therapy has improved survival for those living with HIV infection, with life expectancy now approaching that of uninfected people. Consequently, this patient population is living long enough to develop different types of cancer. At the 23rd NCCN Annual Conference, Gita Suneja, MD, MSHP, presented the debut of the new NCCN Guidelines for Cancer in People Living With HIV, discussing the intersection between cancer and HIV infection, the cancers most likely to develop in this group of patients, and the importance of oncologists working in conjunction with HIV specialists to render the most appropriate and individualized care.
\end{abstract}

J Natl Compr Canc Netw 2018;16(5.5):597-599 doi: 10.6004/jncen.2018.0033

\begin{abstract}
"HIV IV status alone should not be used for cancer treatment decision-making," emphasized Gita Suneja, MD, MSHP, Associate Professor of Radiation Oncology and Global Health, Duke University School of Medicine, and Co-Chair of the NCCN Guidelines Panel for Cancer in People Living With HIV. According to the NCCN Guidelines, this patient population, for whom cancer is now a leading cause of death, should receive cancer treatment per standard guidelines, although modifications in antiretroviral therapy (ART) may be needed. Although Dr. Suneja admitted that HIV management during cancer therapy poses many clinical challenges, "Appropriate cancer treatment can successfully be delivered especially when oncologists and HIV specialists work together to optimize care for both diseases."

Dr. Suneja acknowledged a knowledge deficit surrounding the treatment of cancer in patients with HIV, with $70 \%$ of surveyed oncologists stating that sufficient guidelines were not currently available for treating this population. ${ }^{1}$ Consequently, guidance for practicing cli-

\footnotetext{
Presented by Gita Suneja, MD, MSHP, Department of Radiation Oncology and Duke Global Health Institute, Duke University School of Medicine, Durham, North Carolina.

Dr. Suneja has disclosed that she has no financial interests, arrangements, affiliations, or commercial interests with the manufacturers of any products discussed in this article or their competitors.

Correspondence: Gita Suneja, MD, MSHP, Department of Radiation Oncology and Duke Global Health Institute, Duke University Schoo of Medicine, 30 Duke Medicine Circle, Box 3085, Durham, NC 27710. Email: gita.suneja@icloud.com
}

nicians on how to manage patients with both cancer and HIV infection was sorely needed, thus the inaugural NCCN Guidelines were developed.

Treating patients with cancer who are HIV-positive is complex and requires input from multiple clinicians. Dr. Suneja referenced a survey of 500 medical and radiation oncologists across the United States, ${ }^{1}$ which found that " $20 \%$ to $25 \%$ said they would not offer standard cancer therapy to people living with HIV,... [yet] $45 \%$ said they rarely or never discussed a management plan with an HIV specialist."

\section{Intersection of HIV and Cancer}

People living with HIV have a higher incidence of many cancers compared with the general population, ${ }^{2}$ with multiple contributing factors, including a higher chance of coinfection with oncogenic viruses, such as human papillomavirus (HPV) and human herpesvirus 8 (HHV-8), and a higher prevalence of smoking. ${ }^{3}$

"People living with HIV are less likely to receive cancer treatment compared with uninfected people,"4 said Dr. Suneja, shedding further light on cancer care disparities for those with HIV. Dr. Suneja also noted that "People living with HIV were previously excluded from clinical trials just by virtue of having HIV infection, and that's in part why this knowledge deficit exists." ${ }^{, 6} 6$ For instance, in a 2017 report which examined $>75$ studies, 65 had a specific HIV exclusion, which has undoubtedly 
Suneja

driven disparities in treatment for this unique patient population. ${ }^{5,6}$

\section{AIDS-Defining and Non-AIDS-Defining Malignancies}

Dr. Suneja briefly discussed 2 AIDS-defining cancers (Kaposi sarcoma [KS] and cervical cancer), as well as several non-AIDS-defining malignancies (anal cancer, non-small cell lung cancer, and Hodgkin lymphoma [HL]).

\section{Kaposi Sarcoma}

As one of the first cancers shown to be linked to HIV infection, KS has its own set of newly developed NCCN Guidelines (AIDS-Related KS) separate from the NCCN Guidelines for Cancer in People Living With HIV. Although the risk of developing KS has declined significantly (90\%) with the use of ART, those living with HIV infection are still far more likely to develop KS than uninfected people, revealed Dr. Suneja. HHV-8 is the etiologic infection that underlies KS, and individual lesions may be distinct clones that arise from persistent immunosuppression and HHV-8 infection, which makes treatment of the existing disease tricky because it may not prevent the development of future lesions. Based on the algorithm, ART therapy is among the appropriate first-line options for those with symptomatic disease. "ART therapy is really the backbone of treatment for KS," noted Dr. Suneja. She also mentioned the importance of watching for immune reconstitution inflammatory syndrome, which can be a life-threatening condition.

\section{Cervical Cancer}

The risk of cervical cancer is approximately 3 to 5 times higher in people living with HIV than in the general population, and persistent HPV infection is deemed the etiologic agent. "It is more of a problem in developing countries, because in the United States, we screen for cervical cancer and the US HIV population is largely male," Dr. Suneja explained. The guidelines recommend that women living with HIV and cervical cancer be treated according to the NCCN Guidelines for Cervical Cancer, including the use of concurrent chemotherapy for those receiving definitive radiation treatment.

\section{Anal Cancer}

The risk of developing anal cancer in those with HIV infection is significantly higher than for other types of AIDS-related cancers. The guidelines recommend that those living with HIV infection and anal cancer be treated in concordance with the NCCN Guidelines for Anal Carcinoma. Dr. Suneja pointed out there should be more frequent posttreatment surveillance in the HIV population, with anoscopy every 3 to 6 months for 3 years.

\section{Lung Cancer}

Lung cancer is the most common non-AIDS-defining cancer in people living with HIV infection, with an estimated prevalence 2 to 5 times higher than in the HIV-negative population. At this time, there are no different screening guidelines for lung cancer in HIV populations, but Dr. Suneja suggested that ongoing studies may elucidate alternate screening recommendations for people with HIV in the future. Currently, patients with HIV infection at risk for lung cancer should be screened according to the NCCN Guidelines for Lung Cancer Screening. Lung cancer treatment for those with HIV should parallel the recommendations in the NCCN Guidelines for Non-Small Cell Lung Cancer. Dr. Suneja also noted the importance of smoking cessation in this patient population.

\section{Hodgkin Lymphoma}

$\mathrm{HL}$ is a common type of cancer found in HIV populations, with a 5 to 14 times higher risk of development than the general population. One major distinction for people with HL and an HIV infection, according to Dr. Suneja, is that nearly $90 \%$ of cases are Epstein-Barr virus-associated and that those with HIV infection often are found to have more advanced disease, including B symptoms and bone marrow involvement. Dr. Suneja emphasized that B symptoms should prompt a workup for opportunistic infection, especially if CD4-positive T-cell counts are low.

The ABVD regimen (doxorubicin, bleomycin, vinblastine, and dacarbazine) is recommended for treating HL in people with HIV infection. However, dose reductions may be required for prolonged severe neutropenia. ${ }^{7}$ In this population, growth factors may be required for those with a low CD4-positive T-cell count and prolonged neutropenia, according to Dr. Suneja. Although it is recommended, PET/ CT-guided therapy may be challenging in HIV-positive populations due to potential confounders, such as enlarged or metabolically active nodes due to in- 


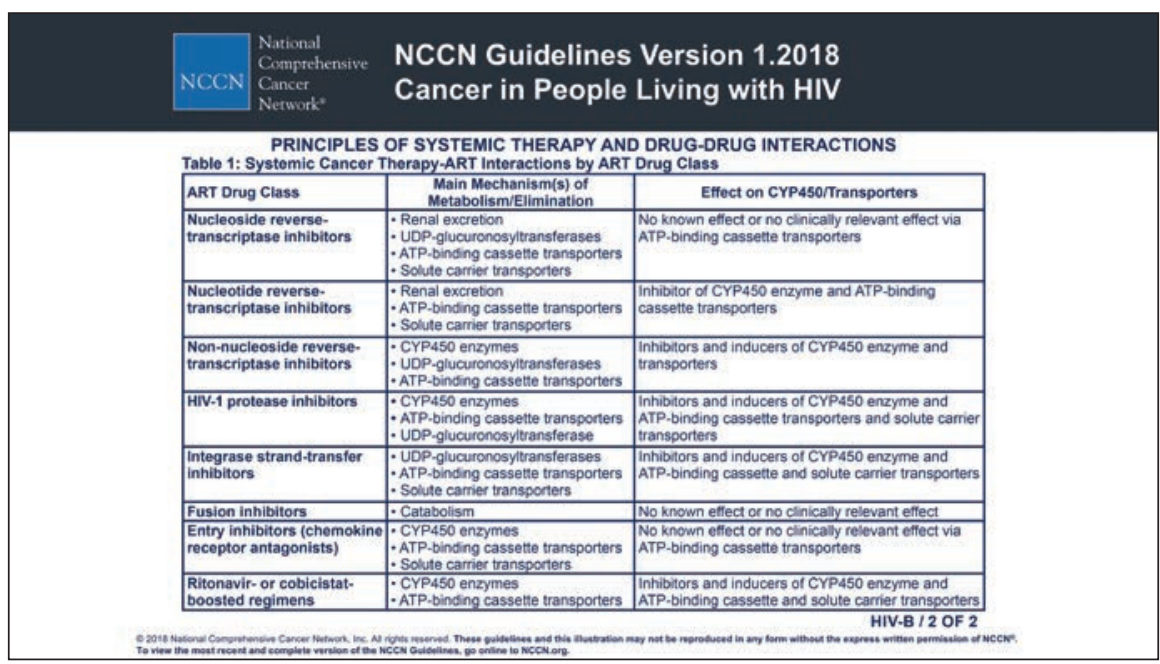

Figure 1. Principles of systemic therapy and drug-drug interactions table from the NCCN Guidelines for Cancer in People Living with HIV.

fection, HIV viremia, or other causes. Autologous stem cell transplant has also been determined to be a safe and effective treatment for those with recurrent or relapsed Hodgkin lymphoma. ${ }^{7}$ In patients with a CD4-positive T-cell count of $<200$ cells/mcL, clinicians should consider prophylactic antibiotics for gram-negative bacteria and Pneumocystis jiroveci pneumonia.

\section{HIV Management in Patients Receiving Cancer Therapy}

"All people with HIV and cancer should be comanaged by an oncologist and an HIV specialist," emphasized Dr. Suneja. HIV therapy should be continued during cancer treatment because it results in better tolerance of cancer treatment, higher response rates, and improved survival. However, modifications to HIV therapy may be required to avoid drug-drug interactions or overlapping toxicities. More frequent CD4-positive T-cell counts and viral load testing should be performed during cancer treatment (once a month for the first 3 months then every 3 months thereafter) due to potential drug interactions that may make ART less effective.

For this patient population, there are unique considerations when selecting drugs for treatment. Dr. Suneja noted the possibility of drug-drug interactions and potential overlapping toxicities, suggesting that cancer therapy, supportive care medications, and ART should be reviewed by an oncologist, an HIV specialist, and a pharmacist. The NCCN Guidelines provide a useful table outlining drug-drug interactions by ART drug class, as well as potential clinically significant pharmacokinetic interactions (Figure 1).

In the guidelines, sections on Principles of Imaging, Principles of Radiation Therapy, and Principles of Surgery are included to guide treatment decisions. In addition, the NCCN Guidelines also offer a section on Principles of Supportive Care, "to ensure that treatment toxicity is minimized," said Dr. Suneja.

\section{References}

1. Suneja G, Boyer M, Yehia BR, et al. Cancer treatment in patients with HIV infection and non-AIDS-defining cancers: a survey of US oncologists. J Oncol Pract 2015;11:e380-387.

2. Engels EA, Pfeiffer RM, Goedert JJ, et al. Trends in cancer risk among people with AIDS in the United States 1980-2002. AIDS 2006;20:16451654.

3. Robbins HA, Pfeiffer RM, Shiels MS, et al. Excess cancers among HIV-infected people in the United States. J Natl Cancer Inst 2015;107:pii: dju503.

4. Suneja G, Shiels MS, Melville SK, et al. Disparities in the treatment and outcomes of lung cancer among HIV-infected individuals. AIDS 2013;27:459-468.

5. Persad GC, Little RF, Grady C. Including persons with HIV infection in cancer clinical trials. J Clin Oncol 2008;26:1027-1032.

6. Uldrick TS, Ison G, Rudek MA, et al. Modernizing clinical trial eligibility criteria: recommendations of the American Society of Clinical Oncology-Friends of Cancer Research HIV Working Group. J Clin Oncol 2017;35:3774-3780.

7. Spitzer TR, Ambinder RF, Lee JY, et al. Dose-reduced busulfan, cyclophosphamide, and autologous stem cell transplantation for human immunodeficiency virus-associated lymphoma: AIDS Malignancy Consortium study 020. Biol Blood Marrow Transplant 2008;14:59-66. 